\section{THE EMERGENCY TREATMENT OF WOUNDS.} LIEUT.-Colonel A. J. HULL, F.R.C.S., R.A.M.C.

IT has been abundantly proved by the experience of war that wounds treated by exeision heal in many cases by primary union. Further, the fact is established that.even when primary union does not occur the more serious types of infected wounds are avoided by this measure, provided the wound is so treated at a suitable time. Anatomical considerations, of course, prevent complete excisions in many cases.

The successful treatment of wounds depends upon the stage at which the wound comes under treatment. Wounds, fortunately, pass through a pre-inflammatory stage, and during this stage it is possible to remove the infected tissues, leaving a non-infected wound. Carrel has shown that a wound passes through a fairly definite chronological sequence. During the first few hours follow. ing its infliction a wound is comparatively clean, judged by bacteriological tests. The smears are usually negative. The organisms have not had sufficient time to multiply in order to appear in the secretions. At the end of five or six hours organisms are frequently found in smears, and ifter twelve hours the wound has commenced to react. Polymorphs appear in large numbers, and bacteriological examination shows a large number of organisms; moreover, the organisms have now spread to the depths of the wound and abound in the particles of fractured bone. It follows that during the first twenty-four hours the wound is amenable to surgical treatment, and after that period an inflammatory stage appears, diuxing which the results of excision have been very much less satisfactory.

The ideal treatment for infected gunshot wounds is to excise them during the pre-inflammatory stage-that is, within twenty-four hours of the infliction. The exigencies of war will more often than not make this impossible. 'The question therefore arises whether it is possible to prolong the pre-inflammatory stage.

Several observers working with flavine have recorded the appearance of the wounds. which are described as in statu quo. There appears to be suppression of cuppura. tion and inflammatory reaction during certain stages of treatment. It may be possible to make use of this or a similar reagent in order to prolong the pre-inflammatory stage sufficiently to allow surgical technique to be employed during the optimum period.

The only treatment which a wound at present receives in war previous to being excised or otherwise dealt with at a casualty clearing station is the application of a first field dressing. It is true that this statement requires modification, inasmuch as it may be injuriously redressed on several occasions, thus delaying the cleansing operation; but the depths of the wound, the shattered fragments of a fractured bone, the parts that really matter, receive no treatment.

In fact, in spite of the adrances in surgery, particularly in the treatment of wounds, the emergency treatment is no more efficacious than it was in the days of the Trojan wars. The dressings applied to wounds before the arrival at a casualty clearing station have no influenoe whatever unon the infection in the depths of the wounds. Improved results of the treatment of wounds can only be achieved by some method of either excising the wound luring the pre-inflammatory stage or by prolonging that itage, and research in the latter direction appears to be inost desirable.

'The old first field dressing appears to be totally inade. quate. It is suggested that, as soon as practicable after the infliction of a wound, it should be instilled with some non-irritating antiseptic, in order to inhibit the growth of unicro-organisms. Within the first few hours, unless septic foreign matter remains in the depths of the wound, pathogenie organisms are found to be few in numbers and confined to the surface of the wound.

When septic foreign bodies are retained in a wound, the growth of organisms would be enormously retarded by the presence of an inhibiting antiseptic. Septic eloth would become soaked with the reagent injected, and would become comparatively innocnous. Excision of the wounds would be carried out in due course with greatly enhanced likelihood of primary union.
Several reagents which are non-irritating inhibitors of septic organisms are available. Flavine in 1 in 1,000 solution or dichloramine- $T$ would appear to be suitable anti. septics for the purpose. Brilliant green is frequently used (as recommended by Captain Hey) as an antiseptic stain. The wound is filled with brilliant green, which acts as a guide to infected tissue, previous to the excision of the septic area. This reagent would fulfil a double purpose if instilled into the wound at an early stage, by acting as an inhibitor of sepsis, and a guide to the septic tissue. Eusol and Dakin solutions would be too evanescent and too transient in their action for this purpose-as, in fact, they are for any wound treatment during transport.

If a 5 per cent. solution of salt be combined with the antiseptic reagent, the micro-organisms will not only be inhibited in the wound, but an outpouring stream of seruin will be produced which will carry infection from the wound, and prevent the ingress of organisms.

The technique of wound instillation is of the simplest possible description. The chosen solution is instilled by means of a fine glass nozzle attached to a Higginson's syringe, care being taken to inject fluid into the depths of the wonnd. This treatment is certainly not beyond the scope of an advanced dressing station.

There is also indication for the use of this method in casualty clearing stations. In times of pressure a patient may have to wait a day or more before an operation can be performed. The preliminary instillation of the wound with an inhibiting antiseptic solution may make the difference of the wound being operated upon in the pre-inflammatory stage, or when suppuration has com. menced. Still more desirable is such treatment when it is necessary to transfer a patient to the base from casualty clearing stations without operation.

I am not at present prepared to recommend the indiscriminate irrigation of wounds of the head and trunk as a preliminary measure, particularly if performed by unskilled hands, but I think its use in the case of wounds of the extremities, particularly compound fractures, is strongly indicated.

The use of preliminary instillation may be objected to on the grounds that the nozzle may introduce infection and that the injection of fluid may spread sepsis. A glass nozzle is easily sterilized between each injection, even at an advanced dressing station, and it is extremely doubtful if such sterilization is necessary. The injection of antiseptic fluid will not spread infection beyond the original limits of the wound, which would become infected in any case. Carrel made use of the instillation treatment in the later treatment of wounds, particularly after wound excision.

By beginning wound instillation within an hour or so we are treating a wound which is bacteriologically as sterile as an excised wound, and but for the tissue necrosis which follows gunshat wounds the early instillation of a suitable antiseptic might replace wound excision.

REFERENCEs.

Carrel and Dehelly, The Treatment of Infected Wounds, 1917. Fiesinger, La pratique de la chiruroie de guerre. 1916.

TEMPORARY CAECOSTOMY IN RESECTION OF

THE DISTAL PORTION OF THE COLON FOR NON-OBSTRUCTIVE CONDITIONS.

\section{BY}

GORDON TAYLOR, M.A.ABERD., M.S., B.Sc.LoNd., F.R.C.S., MAJOR R.A.M.C.(T.C.)

BENIOR ABgISTANT SURGKON, MIDDLESEX HOSPITAL, ETC.

This note has been written to draw attention to a little surgical manœurre which has been of service to me in cases of resection of portions of the distal half of the large intestine. This step in the surgical technique of excision of any portion of the large bowel betwoen the splenic flexure and the sigmoid colon I learnt in the course of conversation with a very distinguished Scottish surgeon who employed it, but in France I have met with very fow surgeons who appeared to be familiar with the procedure, either in theory or in practice. It is of course established now that excision of portions of the large intestine for gunshot injury in the "fon ward area" is only 